\title{
Relación entre la exposición a pesticidas y las enfermedades mentales: Una revisión sistemática
}

\author{
Relationship between pesticide exposure and mental illness: A systematic \\ review
}

\author{
Nuria García-Martínez ${ }^{1 *}$, Inmaculada Navarro-González², Pedro Andreo-Martínez ${ }^{3}$
}

\begin{abstract}
Citación/Citation: García-Martínez N, NavarroGonzález I, Andreo-Martínez P. Rev Dis Cli Neuro. 2021; (8)1: 14-27. Available from:

https://doi.org/10.14198/DCN.19700
\end{abstract}

Recibido/Received: $16 / 04 / 2021$

Aceptado/Accepted: 19/04/2021

Financiación/Funding: Este estudio no ha recibido financiación.

Conflicto de intereses/Conflicts of Interest: Los autores declaran no tener conflicto de intereses.

@ 2021 Nuria García-Martínez, Inmaculada
Navarro-González y Pedro Andreo-Martínez

Licencia/Licence: This work is licensed under a Creative Commons Attribution 4.0 International License (CC BY 4.0).

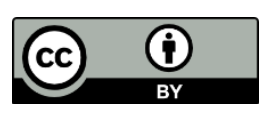

Editorial: Universidad de Alicante (España) Autores/Authors:

\footnotetext{
${ }^{1}$ Departamento de Química Agrícola, Geología y Edafología. Universidad de Murcia. España, https://orcid.org/0000-0001-9028-231X

2 Área Científica y Técnica de Investigación (ACTI) Sección de Biología Molecular. Universidad de Murcia. España, https://orcid.org/0000-00025894-6400

${ }^{3}$ Departamento de Química Agrícola, Geología y Edafología. Universidad de Murcia. España, https://orcid.org/0000-0001-6535-5492

* Contact for correspondence: n.garciamartinez@um.es
}

Resumen: Este trabajo realiza una revisión sistemática sobre la relación existente entre la exposición a residuos de pesticidas y la presencia de enfermedades o trastornos mentales. Los pesticidas son sustancias químicas utilizadas en la inmensa mayoría de los campos agrícolas para prevenir o eliminar las plagas que amenazan a los cultivos. Sin embargo, su uso puede implicar serios problemas para la salud de las personas como enfermedades pulmonares, cáncer, enfermedades metabólicas, problemas de infertilidad e incluso trastornos psicopatológicos. La metodología para realizar este estudio de revisión siguió las indicaciones marcadas por el protocolo PRISMA, encontrándose un total de cuatro artículos originales tras aplicar los criterios de exclusión e inclusión establecidos. Tres artículos estudiaron la relación exposición a residuos pesticidas enfermedades mentales en sujetos con características diferentes y con niveles de exposición variables. Única-mente un artículo empleó ratones sometidos a diferentes dosis de pesticidas como modelo para evaluar su efecto sobre el comportamiento de estos animales. De los resultados encontrados por los diferentes artículos se puede deducir que la exposición a residuos de pesticidas tiene claros efectos sobre la salud mental de individuos altamente susceptibles a la presencia de contaminantes como mujeres lactantes y recién nacidos; así como personas sometidas a elevados niveles de pesticidas como formuladores y aplicadores agrícolas o personas residentes en las cercanías de campos agrícolas. Por otro lado, futuras investigaciones sobre este tema, basadas en modelos animales y estudios poblacionales, son necesarias para establecer conexiones realistas entre los trastornos mentales y los diferentes tipos de pesticidas usados actualmente en los campos agrícolas.

Palabras clave: Enfermedades mentales; Enzima acetilcolinesterasa; Hormonas tiroideas; Pesticida; Trastornos neuropsiquiátricos. 


\begin{abstract}
This work carries out a systematic review on the relationship between exposure to pesticide residues and the presence of mental illnesses or disorders. Pesticides are chemical compounds used in the vast majority of agricultural fields to prevent or eliminate pests that threaten crops. However, its use can involve serious human health problems such as lung diseases, cancer, metabolic diseases, infertility problems and even psychopathological disorders. The methodology for conducting this review study followed the indications set by the PRISMA protocol, finding a total of four original articles after applying the established exclusion and inclusion criteria. Three articles studied the relationship between pesticide residues exposure - mental illnesses in subjects with different characteristics and variable levels of exposure. Only one article used mice subjected to different doses of pesticides as a model to evaluate their effect on the behavior of these animals. From the results found by the different articles it can be deduced that exposure to pesticide residues has clear effects on the mental health of individuals highly susceptible to the presence of contaminants such as lactating women and newborns; as well as people subjected to high levels of pesticides such as agricultural formulators and applicators or people residing in the vicinity of agricultural fields. On the other hand, future researches on this topic, based on animal models and population studies, are necessary to establish realistic connections between mental disorders and the different types of pesticides currently used in agricultural fields.
\end{abstract}

Keywords: Acetylcholinesterase enzyme; Mental illness; Neuropsychiatric disorders; Pesticide; Thyroid hormones.

\title{
1. Introducción
}

Las plagas comprometen la calidad y disponibilidad de nuestros alimentos, siendo necesaria la aplicación de pesticidas (herbicidas, fungicidas, plaguicidas e insecticidas) para prevenir, destruir, repeler o mitigar su acción, beneficiando la agricultura y la salud pública. Los pesticidas son las únicas sustancias que se liberan intencionalmente en nuestro medio ambiente para matar seres vivos y son utilizados ampliamente en todos los campos de cultivo del mundo (2,7 millones de toneladas en 2011-2012). Sin embargo, su aplicación, además del impacto ambiental sobre la flora y fauna, incluidos insectos beneficiosos, polinizadores, anfibios y aves, puede suponer riesgos para la salud humana. De hecho, numerosos estudios han demostrado que existe una estrecha asociación entre la exposición a pesticidas y el desarrollo de enfermedades como el cáncer, asma, bronquitis, diabetes, obesidad, infertilidad, defectos de nacimiento, enfermedades neurodegenerativas como el Alzheimer, Parkinson y esclerosis lateral amiotrófica, y otros trastornos como el trastorno por déficit de atención con hiperactividad (TDHA), autismo, depresión, y otros desórdenes del comportamiento. La mayoría de los trastornos son inducidos por insecticidas y herbicidas, sobre todo organofosforados, organoclorados, ácidos fenoxiacéticos y compuestos de triazinas [1-4].

La neurotoxicidad de estas sustancias depende en gran medida del grado de exposición, el cual puede ocurrir a través de la producción, el transporte, la entrega y la aplicación de plaguicidas, la residencia en lugares con alto contenido en residuos de plaguicidas y la circulación y acumulación de éstos en la cadena alimentaria. Otros factores importantes son la tipología de la sustancia química y las características del 
individuo, afectando no sólo a adultos, sino a niños y fetos. De hecho, se han encontrado que diferentes disruptores endocrinos, como residuos de plaguicidas organoclorados ( $\alpha$-clordano, $\gamma$-clordano, heptacloro, diclorodifenildicloroetileno (DDE), diclorodifenil-tricloroetano (DDT) y plaguicidas organofosforados (clorpirifos y clorfenvinfos), pueden ser responsables de casos de muerte intrauterina inexplicable y casos de síndrome de muerte súbita del lactante en zonas dedicadas a la agricultura intensiva; e incluso se han encontrado estos contaminantes en cordones umbilicales de fetos expuestos a los mismos por la dieta de la madre. Por tanto, queda demostrada la capacidad de algunos residuos de plaguicidas para atravesar la barrera placentaria y llegar a los cerebros fetales y neonatales [5, 6]. Además, los niños son más vulnerables que los adultos al impacto de un nivel equivalente de exposición a estos químicos, debido al estado inicial de desarrollo de órganos, la falta de madurez fisiológica, el menor peso corporal, la mayor ingesta de comida y bebida, y la mayor tasa respiratoria. Por ello, a cortas edades, el impacto de los residuos de pesticidas puede ser mayor, lo que puede originar, en ciertos casos, problemas en el desarrollo neurológico y trastornos neuropsiquiátricos que arrastrarán en la edad adulta [7].

Lo que es evidente, a la vista de las últimas investigaciones desarrolladas sobre los posibles factores exógenos promotores de enfermedades mentales, es que determinados residuos de pesticidas son potencialmente inductores de algunas enfermedades y trastornos mentales. El mecanismo de acción sobre el cerebro depende de la tipología del químico. En el caso de los pesticidas organofosforados, producen efectos tóxicos sistémicos como consecuencia de un doble mecanismo: por un lado, al atacar el sistema nervioso inhibiendo el neurotransmisor acetilcolinesterasa, provocando síntomas de hiperestimulación colinérgica, lo que deriva en déficits en el desarrollo neurológico; y por otro, mediante la interrupción generalizada de la replicación y diferenciación de las células neurales, la axonogénesis y la sinaptogénesis, y la función sináptica. Todos estos procesos son esenciales para las tareas cognitivas y conductuales [7]. El mecanismo de actuación de los pesticidas organoclorados se basa, de forma genérica, en la alteración de proteínas como enzimas antioxidantes, enzimas metabólicas (como la acetilcolinesterasa), transportadores de neurotransmisores como la dopamina o canales iónicos como las bombas de $\mathrm{Mg}^{2+}, \mathrm{Na}^{+} / \mathrm{K}^{+} \mathrm{y} \mathrm{Ca}^{2+}$. Todas estas alteraciones afectan al sistema nervioso central generando déficits de aprendizaje y de memoria, problemas locomotores y problemas de comportamiento [8, 9].

En la literatura científica existen gran cantidad de revisiones descriptivas sobre la relación entre la exposición a pesticidas y enfermedades metabólicas, pulmonares y cancerígenas [10-12]. Sin embargo, las revisiones que se centran en la vinculación de las enfermedades mentales y la exposición a estos químicos son escasas y descriptivas $[4,7,13,14]$. Bajo el conocimiento de los autores, no existen en la literatura científica revisiones sistemáticas sobre las implicaciones de la exposición a residuos pesticidas y las enfermedades mentales.

\section{Objetivo}

El objetivo de este estudio es proporcionar la información existente sobre la relación entre la exposición a residuos de pesticidas y las enfermedades mentales utilizando como herramienta el protocolo PRISMA. 


\section{Método}

La metodología de búsqueda bibliográfica para obtener las publicaciones científicas más relevantes sobre el objetivo de esta revisión sistemática siguió las indicaciones propuestas por el protocolo PRISMA [15]. El periodo de tiempo elegido fue desde que existen registros de publicaciones en las bases de datos hasta el 13 de abril de 2021. Las bases de datos consultadas fueron Web of Science, Scopus, Science Database y PubMed, utilizando la cadena de booleanos "pesticide residue*" AND "mental illness" OR "mental disease". Las opciones de búsqueda en las diferentes bases de datos fueron: "theme" en la Web of Science, "Article title, Abstract, Keyword" en Scopus y "todos los campos" en Science Database y PubMed.

Los criterios de inclusión fueron: estudios científicos sobre la relación entre la exposición a residuos pesticidas y las enfermedades mentales y artículos publicados desde que existen registros en las bases de datos hasta el 13 de abril de 2021. Los criterios de exclusión fueron: revisiones, disertaciones en congresos, libros y capítulos de libro, material editorial, cartas al editor, y artículos que no estudien la relación entre las enfermedades mentales y los pesticidas. Los resultados arrojados por las bases de datos se cruzaron con el programa informático EndNote X9 para detectar posibles duplicados. El equipo revisor estuvo formado por los autores Pedro Andreo-Martínez y Nuria García-Martínez.

Una vez obtenido el número de artículos incluidos para esta revisión, se inició la evaluación de la calidad de los mismos, que consistió en la valoración de los riesgos de sesgo de cada uno de los artículos, siguiendo el modelo publicado en estudios previos [16, 17].

\section{Resultados}

La Figura 1 muestra el diagrama de flujo PRISMA que se ha seguido para seleccionar los artículos para la presente revisión sistemática. La base de datos Web of Science arrojó 1 resultado, mientras que en Scopus se obtuvieron un total de 8 resultados. No se obtuvieron resultados en las búsquedas realizadas en las bases de datos PubMed y Science Database y tampoco se encontraron trabajos duplicados. De las 9 referencias obtenidas, 3 eran revisiones y 1 era una carta al editor, por lo que se revisaron un total de 5 artículos a texto completo para evaluar su elegibilidad. De esos 5 trabajos, se excluyó un artículo que abordaba cuestiones de política y salud pública y se centraba en aspectos regulatorios sobre pesticidas, no incluyendo investigaciones sobre el objeto de estudio. Finalmente se encontró que 4 artículos cumplieron con los criterios de inclusión de la presente revisión sistemática.

Los 4 artículos seleccionados fueron publicados en 4 revistas diferentes: Toxicology Letters, International Journal of Environmental Research and Public Health, International Journal of Environmental Health Research y Chemosphere. De acuerdo con el autor de correspondencia, un artículo (25\%) fue publicado en Egipto, uno (25\%) en China, uno (25\%) en Estados Unidos, y uno (25\%) en Turquía. Los artículos fueron publicados entre 1999 y 2020. 
Figura 1. Diagrama de flujo de los artículos seleccionados.

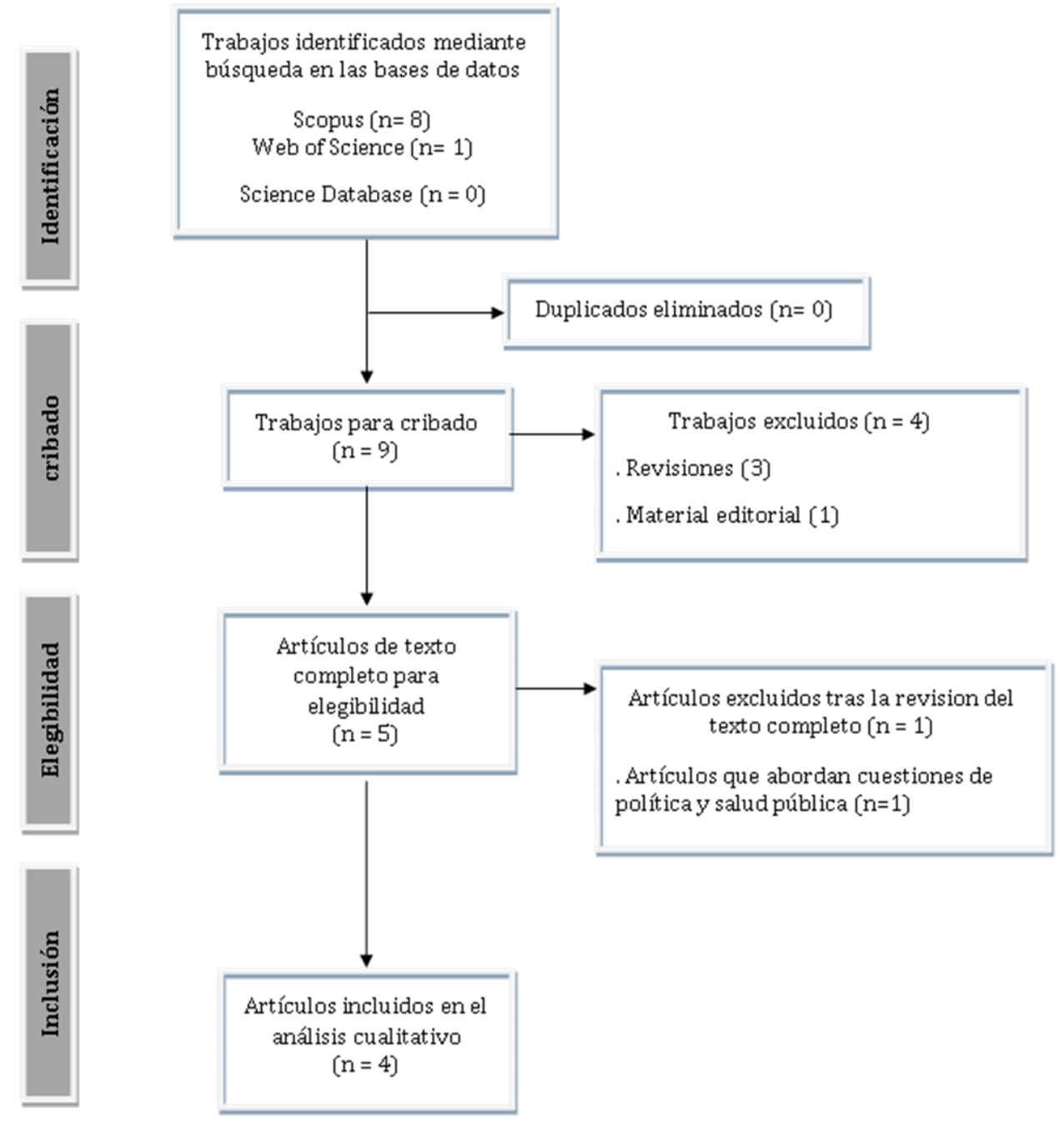

En la Tabla 1 se indican los estudios seleccionados y los principales resultados de las investigaciones sobre la relación exposición a pesticidas - enfermedades mentales. El estudio realizado por Amr [18] se basó en la detección de problemas de salud, principalmente en la presencia de manifestaciones neuropsiquiátricas, en formuladores y aplicadores de pesticidas. En este estudio también se evaluó la presencia de residuos de pesticidas organoclorados en diferentes productos lácteos. M Khan, Karnati [19] evaluaron las posibles enfermedades y trastornos mentales de adultos que viven en zonas cercanas a campos agrícolas donde se aplican pesticidas para el control de plagas, con el fin de establecer una relación causa- efecto. El trabajo realizado por Yalçın, Örün [20] investigó la presencia de pesticidas organoclorados en la leche materna, sus efectos en las características materno-infantiles y posibles implicaciones en las psicopatologías maternas. Por último, Zhang, Liu [21] estudiaron los posibles efectos de la deltametrina, un insecticida de baja toxicidad y altamente empleado, en el eje hipotalámico-pituitario-tiroideo y el comportamiento en ratones adolescentes. Mientras que este último estudio se realizó utilizando ratones como modelo animal, el resto de investigaciones se realizaron sobre población expuesta, de un modo $\mathrm{u}$ otro, a residuos pesticidas. 
Tabla 1. Objetivo, muestra objeto de estudio, pesticidas analizados, diseño experimental, determinaciones analíticas y principales resultados encontrados en los 4 estudios seleccionados en la presente revisión sistemática.

\begin{tabular}{lllllll}
\hline $\begin{array}{l}\text { Referenc } \\
\text { ia }\end{array}$ & Objetivo & $\begin{array}{l}\text { Muestra } \\
\text { objeto de } \\
\text { estudio }\end{array}$ & $\begin{array}{l}\text { Pesticidas } \\
\text { estudiados }\end{array}$ & $\begin{array}{l}\text { Diseño } \\
\text { experimental }\end{array}$ & $\begin{array}{l}\text { Determinacione } \\
\text { s analíticas }\end{array}$ & $\begin{array}{l}\text { Principales } \\
\text { resultados }\end{array}$ \\
\hline
\end{tabular}

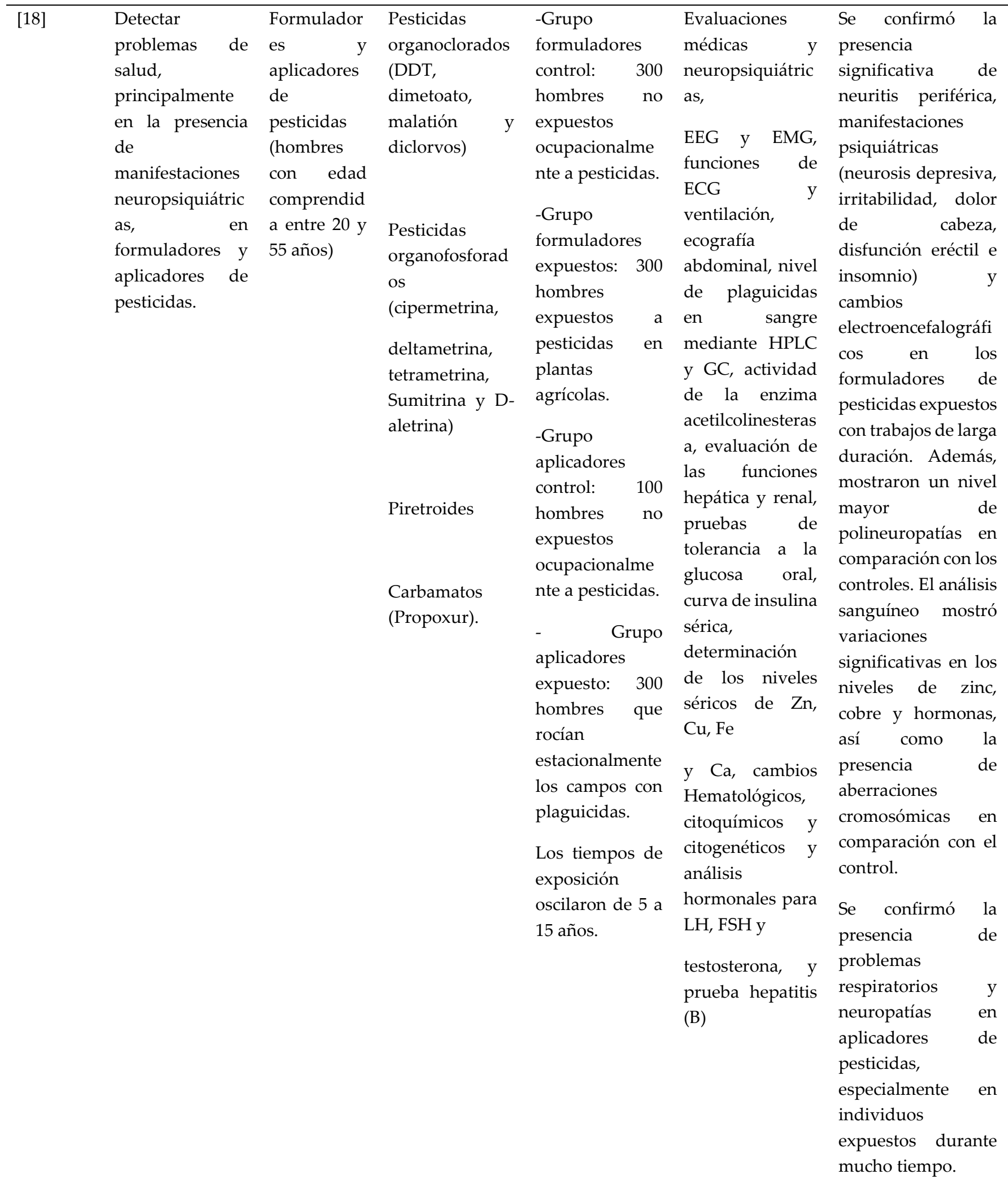


[20]

$\begin{array}{lll}\begin{array}{l}\text { Investigar la } \\ \text { relación entre la lactantes }\end{array} & \alpha-\mathrm{HCH} \\ \begin{array}{l}\text { presencia de sus bebés. } \\ \text { pesticidas } \\ \text { organoclorados } \\ \text { en la leche }\end{array} & \beta-\mathrm{HCH} \\ \begin{array}{l}\text { materna y las } \\ \text { características } \\ \text { materno- } \\ \text { infantiles y las } \\ \text { psicopatologías } \\ \text { maternas. }\end{array} & \text { Aldrin } \\ & \text { Dieldrin } \\ & \text { Epóxido } \\ & & \alpha \text {-Endosulfan } \\ & & \beta \text {-Endosulfan } \\ & & \text { Trans-clordano } \\ & & \text { Cis-clordano } \\ & & \text { DDT }\end{array}$

[19]

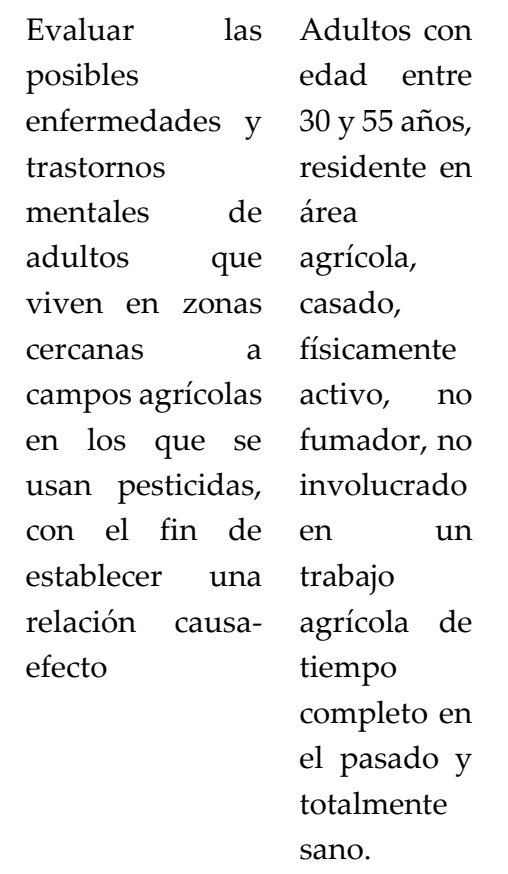

- Se recolectaron

75 muestras de

leche maternal

humana en el

octavo mes

posparto.

-Mediante

cuestionarios a

la madre se

obtuvieron las

características

del bebé y la madre.

DDT

Carbamato
Organofosforad
os

Oxadiazon

Piretroide

Zeta-

cipermetrina

Grupo control: residencia

alejada

campos

tratados

pesticidas $(>200$

$\mathrm{m})$.

Grupo

expuesto:

residencia

cercana

campos

tratados con

pesticidas $(<200$

$\mathrm{m})$.

Ambos grupos

se sometieron a

cuestionarios $\mathrm{y}$ análisis.
-Evaluación
posparto de
madres y bebes y
estudio
psicológico en
los 8 meses
postparto
mediante BSI.
DDT,
$\beta-\mathrm{HCH}$,
aldrina y heptacloro
fueron los pesticidas
que se encontraron
en mayor
proporción en las muestras de leche materna.

-Medidas

antropométricas

del bebe (peso,

altura $y$

diámetro de

cabeza) en el

octavo mes

posparto.

-Análisis

de

residuos

de

plaguicidas

organoclorados

de las muestras

de

mediante leche

cromatografía

gaseosa.

fía

Mujeres lactantes con niveles elevados de DDT y beta-HCH en la leche materna tuvieron bebés con un perímetro cefálico pequeño.

Se encontraron correlaciones positivas entre las madres con niveles altos de epóxido de heptacloro y los índices BSI, indicando la posible presencia de psicopatologías maternas $\mathrm{y}$ problemas de apego madre-hijo.

Se encontraron diferencias significativas en las puntuaciones medias de la prueba de umbral vibrotáctil y la prueba B de creación de senderos entre los dos grupos, lo que refleja un posible signo de neuropatía periférica y un posible deterioro cognitivo.

Estudio de mercado de No se encontraron plaguicidas de la zona.

-Evaluación neurológica mediante tres no

\section{diferencias}

significativas entre los grupos para otros resultados 


\begin{tabular}{|c|c|}
\hline $\begin{array}{lr}\text { Encontrar los } \\
\text { efectos de la }\end{array}$ & $\begin{array}{l}\text { Ratones } \\
\text { hembra de }\end{array}$ \\
\hline deltametrina & tres \\
\hline sobre el eje & semanas de \\
\hline $\begin{array}{l}\text { hipotalámico- } \\
\text { pituitario- }\end{array}$ & edad \\
\hline $\begin{array}{l}\text { tiroideo y el } \\
\text { comportamiento }\end{array}$ & aclimata \\
\hline $\begin{array}{l}\text { en ratones } \\
\text { adolescentes }\end{array}$ & \\
\hline
\end{tabular}

invasivos: test de

trazado,

medición

umbral

vibrotáctil

medición de la

velocidad de

conducción del

nervio motor

cubital.

- Cuestionario de síntomas

neurológicos.

Deltametrina La DM se disolvió en aceite de maíz.

- Grupo control (1): ratones con aceite de maíz suministrada por sonda.

-Grupos de estudio ratones con DM suministrada por sonda a dosis de 3, 6 o 12 $\mathrm{mg} / \mathrm{Kg}$ por día (durante 28 días)
-Determinación del periodo de celo de los ratones.

-Pruebas de comportamiento : actividad circadiana, actividad locomotora, pruebas de caja clara oscura, construcción de nidos y pruebas de natación forzada.

-Decapitación, recolección de muestras biológicas análisis hormonas séricas, total, y examen histopatológico. neurológicos y de salud mental.

1


comportamiento en los ratones.

Nota: BSI (Brief Symptom Inventory); DDT (diclorodifeniltricloroetano); DM (deltametrina); EEG (electroencephalographic changes); HPLC (cromatografía líquida de alta resolución); GC (cromatografía gaseosa); HCH (hexaclorociclohexano); EEG (electroencefalograma); EMG (electromiograma)

En cuanto al riesgo de sesgo, se consideró que los artículos tenían un riesgo de sesgo alto, moderado o bajo sobre una puntuación de 12. Se encontró un riesgo de sesgo bajo en los 4 artículos seleccionados en la presente revisión sistemática. Los detalles completos de la puntuación en los diferentes tipos de sesgo se recogen en la Tabla 2.

Tabla 2. Revisión de la calidad para cada artículo incluido.

\begin{tabular}{|c|c|c|c|c|}
\hline Artículo & [19] & {$[20]$} & {$[21]$} & {$[18]$} \\
\hline 1. Objetivo claramente establecido & 2 & 2 & 2 & 2 \\
\hline 2. Tamaño del estudio apropiado & 1 & 2 & 1 & 2 \\
\hline 3. Identificación y evaluación de la muestra & 2 & 2 & 2 & 2 \\
\hline 4. Comparabilidad & 2 & 2 & 2 & 2 \\
\hline $\begin{array}{l}\text { 5. Otros sesgos (imposibilidad de generalización de } \\
\text { resultados, datos de exposición previa, etc.) }\end{array}$ & 1 & 2 & 1 & 2 \\
\hline 6. Análisis estadístico adecuado & 2 & 2 & 2 & 2 \\
\hline TOTAL & 10 & 12 & 10 & 12 \\
\hline Riesgo de sesgo & 2 & 0 & 2 & 0 \\
\hline Riesgo de sesgo general & B & B & B & B \\
\hline
\end{tabular}

Por definición, la presente revisión sistemática estuvo limitada por las bases de datos utilizadas, los términos de búsqueda elegidos y los criterios de inclusión / exclusión establecidos. No se pudo realizar un metaanálisis de los datos de los estudios obtenidos debido al pequeño número de estudios [22].

\section{Discusión}

Tres de los artículos encontrados en la presente revisión sistemática estudiaron la relación existente entre la exposición a residuos pesticidas y la presencia de enfermedades mentales o desórdenes neuropsiquiátricos en sujetos con características diferentes y con niveles de exposición variables: individuos con un alto grado de vulnerabilidad a la presencia de pesticidas, como es el caso de madres lactantes y recién nacidos, y sujetos sometidos a medios y elevados niveles de residuos de pesticidas, como 
adultos con residencias cercanas a campos agrícolas y formuladores o aplicadores de pesticidas. Únicamente un artículo empleó ratones sometidos a diferentes dosis de pesticidas como modelo para evaluar su efecto sobre el comportamiento de estos animales.

\subsection{Individuos altamente susceptibles a la presencia de pesticidas: mujeres lactantes y recién nacidos}

Los niños, las mujeres embarazadas, los bebés, los ancianos y las personas con problemas de salud son más susceptibles a los contaminantes y pueden desarrollar efectos adversos más severos que el resto de la población. La elevada lipofilia de la mayoría de los pesticidas, principalmente los organoclorados, hace que se absorban fácilmente por la piel viajando hacia los tejidos por el plasma unido a albúmina y lipoproteínas. Luego, se difunden entre las membranas, se introducen en las células y se acumulan en órganos con niveles altos de grasa, incluidos el hígado, los riñones, el cerebro y el tejido mamario [23].

La leche materna es considerada un alimento completo para los bebés lactantes menores de 6 meses. Sin embargo, contaminantes químicos como los pesticidas organoclorados, debido a su capacidad de persistencia en el medio ambiente y la bioacumulación a lo largo de la cadena alimentaria, son capaces de pasar a la leche materna a través de la sangre y el tejido mamario causando efectos adversos en la salud del lactante (bajo peso al nacer, retardo del crecimiento, retraso en el neurodesarrollo o partos prematuros, entre otros), especialmente en esta etapa crítica de desarrollo, e incluso psicopatologías maternas [20].

La leche materna es una matriz biológica excepcional para investigar, sin necesidad de usar métodos invasivos, la presencia de ciertos contaminantes ambientales ya que proporciona información fiable sobre la exposición de la madre lactante y de su bebé. Además, permite una extracción eficiente de los contaminantes químicos y una sencilla disponibilidad de muestra [20, 24].

Según Yalçın, Örün [20], los pesticidas que se encuentran en mayor proporción en las muestras de leche materna son DDT, $\beta$-hexaclorociclohexano $(\beta-\mathrm{HCH})$, aldrina y heptacloro. Las proporciones en las que se encuentran estos contaminantes varían en función del país o región de residencia de las madres, condición social, número de partos y otras características gestacionales. Los casos de mujeres lactantes con niveles elevados de DDT y beta-HCH en la leche materna estaban relacionados con bebés con un perímetro cefálico pequeño al octavo mes de edad, lo que podría implicar mayores probabilidades de TDAH del niño en el futuro, ya que dicho trastorno se asocia a elevados niveles de $\beta-\mathrm{HCH}$ en la leche materna [25]. Además, se encontraron correlaciones positivas entre las madres con niveles altos de epóxido de heptacloro y los índices BSI (Brief Symptom Inventory), lo que podría indicar la presencia de algunas psicopatologías maternas y problemas de apego madre-hijo.

Uno de los insecticidas más utilizados en el mundo, la deltametrina (DM), es un piretroide capaz de acumularse en tejidos humanos y la leche materna. También es capaz de atravesar la barrera placentaria y llegar al cerebro del feto. La exposición a DM en mamíferos puede generar, además de genotoxicidad, inmunotoxicidad y toxicidad reproductiva, y serios problemas de neurotoxicidad. De hecho, se ha demostrado que la descendencia de ratonas preñadas expuestas a DM, tiene elevadas probabilidades de padecer trastorno por déficit de atención con hiperactividad. Al parecer, el TDHA se debe a déficits dopaminérgicos en el cerebro [26, 27].

Zhang, Liu [21] analizaron los cambios de comportamiento de ratones adolescentes sometidos a diferentes dosis de DM. Los autores observaron que la movilidad de los ratones se incrementaba notablemente con la dosis de DM, lo que implicaba que la exposición al pesticida generaba hiperactividad. Además, los 
ratones expuestos al contaminante creaban nidos con menor cantidad de tejido, lo que sugirió que presentaban déficit de atención. La prueba de natación concluyó que los ratones expuestos, que permanecían más tiempo inmóviles en el agua, presentaban trastornos depresivos. También encontraron una disminución de las hormonas tiroideas, daño en la morfología del tejido tiroideo y disminución de la dopamina. Estas alteraciones generaron anomalías de comportamiento en los ratones. Este estudio confirma los problemas de neurotoxicidad que puede ocasionar la exposición a este pesticida, siendo especialmente preocupante los daños neurológicos que puede generar en neonatos.

\subsection{Individuos sometidos a medios y altos niveles de exposición}

En la agricultura, la exposición directa de los trabajadores a múltiples plaguicidas es innegable y está asociada a enfermedades neurodegenerativas como el Parkinson y resultados neuroconductuales adversos como el deterioro de la memoria visual, fluidez del lenguaje y problemas de atención. La gran incidencia de estos trastornos en este sector (aplicadores, formuladores, recolectores, etc.) se debe al elevado tiempo de exposición, niveles de concentración de estos contaminantes en el ambiente y la falta de conocimiento sobre prácticas de seguridad agrícola, especialmente la falta de uso de equipos de protección individual [19, 28, 29].

Amr [18] confirmó la presencia de neuritis periférica (> $40 \%)$, manifestaciones psiquiátricas (45\%), como la neurosis depresiva, irritabilidad, dolor de cabeza, disfunción eréctil e insomnio, y cambios electroencefalográficos (25\%) en los formuladores de pesticidas. Estos trastornos eran más evidentes en individuos de mayor edad y con trabajos de larga duración. El análisis sanguíneo mostró variaciones significativas en los niveles de zinc, cobre y hormonas, así como la presencia de aberraciones cromosómicas en comparación con el grupo control. Más del 40 \% del grupo expuesto sufrió impotencia como consecuencia de un aumento en el nivel de testosterona causado por los efectos de los pesticidas en el metabolismo de la misma. En los aplicadores de pesticidas también se confirmó la presencia de neuropatías (36\%). Estos trastornos neurológicos podrían deberse al hecho de que los pesticidas organofosforados y los carbamatos impiden la descomposición de la enzima acetilcolinesterasa provocando su acumulación en el cerebro y produciendo alteraciones en el sistema nervioso [30].

La concentración de pesticidas en el aire disminuye a medida que uno se aleja de las tierras de cultivo. Por ello, las personas que viven en zonas rurales cercanas a campos agrícolas tratados con pesticidas también pueden llegar a tener niveles de exposición medios o altos a estos contaminantes, siendo más vulnerables a los mismos los grupos poblacionales más sensibles (niños, ancianos, embarazadas, etc.) [31].

M Khan, Karnati [19] encontraron posibles signos de neuropatía periférica y deterioro cognitivo en adultos cuya residencia estaba en las cercanías de campos de cultivo tratados con pesticidas. Los ensayos en la prueba de umbral vibrotáctil y la prueba de creación de senderos que miden la función nerviosa periférica y la atención visual, respectivamente, (ambas habilidades asociadas con la función del sistema nervioso central), mostraron que las personas que vivían a menos de $200 \mathrm{~m}$ de los campos agrícolas necesitaban un mayor estímulo para la detección de vibraciones, lo que refleja un posible signo de neuropatía periférica. Además, los resultados en la prueba de creación de senderos indicaron un posible deterioro cognitivo y un funcionamiento ejecutivo en general más bajo. Pese a que no se encontraron evidencias de otras anomalías neurológicas y de salud mental, estos resultados pueden indicar posibles efectos a largo plazo de la exposición a pesticidas tanto en el sistema nervioso central como en el periférico. 


\section{Conclusión}

La exposición a residuos de pesticidas tiene claros efectos sobre la salud mental de individuos altamente susceptibles a la presencia de contaminantes como los ancianos, mujeres embarazadas, mujeres lactantes, niños y recién nacidos; así como personas sometidas a elevados niveles de pesticidas como consecuencia de su trabajo (formuladores, aplicadores, recolectores, etc.) o su residencia, cercana a campos agrícolas.

Los efectos neuropsiquiátricos demostrados de los pesticidas son muy variados, y dependen de la tipología y concentración del químico y del individuo afectado. Se han encontrado evidencias de trastornos psicopatológicos maternos y problemas de apego madre-hijo en presencia de exposiciones altas de epóxido de heptacloro. Además, elevados niveles de $\beta-\mathrm{HCH}$ y DM en la leche materna podrían generar problemas de TDAH del niño lactante en el futuro al ser capaces de alterar los niveles de hormonas tiroideas y de dopamina. Un elevado porcentaje de trabajadores altamente expuestos y personas residentes en zonas con elevada concentración de pesticidas presentan trastornos como neuritis periférica, neurosis depresiva, irritabilidad, dolor de cabeza, disfunción eréctil, insomnio, deterioro cognitivo y cambios electroencefalográficos, de forma más acusada en individuos de mayor edad y con trabajos de larga duración. Algunas de las causas de la aparición de estas disfunciones radican en la presencia de aberraciones cromosómicas, alteración de los niveles de hormonas y la modificación de la actividad de la enzima acetilcolinesterasa.

Los estudios realizados hasta la fecha sobre la implicación de los residuos pesticidas en las enfermedades mentales son escasos, y poco particularizados al tipo de químico. Es necesario el desarrollo de más investigaciones sobre la neurotoxicidad de los pesticidas tomando modelos animales para poder especificar los efectos de un contaminante determinado a diferentes niveles de exposición, así como el posible comportamiento sinérgico de varios pesticidas. Los estudios futuros también deberían enfocarse en estudios sobre salud mental en muestras poblacionales suficientemente grandes, con las variabilidades que ello conlleva, expuestas a estos químicos.

\section{Referencias}

1. Requena M, Parrón T, Navarro A, García J, Ventura MI, Hernández AF, et al. Association between environmental exposure to pesticides and epilepsy. Neurotoxicology 2018, 68: 13-18. doi: https://doi.org/10.1016/j.neuro.2018.07.002

2. Sabarwal A, Kumar K, Singh RP. Hazardous effects of chemical pesticides on human health-Cancer and other associated disorders. Environ Toxicol Pharmacol 2018, 63: 103-114. doi: https://doi.org/10.1016/j.etap.2018.08.018

3. Larsen AE, Patton M, Martin EA. High highs and low lows: Elucidating striking seasonal variability in pesticide use and its environmental implications. Sci Total Environ 2019, 651 (Pt 1): 828-837. doi: https://doi.org/10.1016/j.scitotenv.2018.09.206

4. Mostafalou S, Abdollahi M. Pesticides: an update of human exposure and toxicity. Arch Toxicol 2017, 91 (2): 549-599. doi: https://doi.org/10.1007/s00204-016-1849-x

5. Roncati L, Pusiol T, Piscioli F, Lavezzi AM. Neurodevelopmental disorders and pesticide exposure: the northeastern Italian experience. Arch Toxicol 2017, 91 (2): 603-604. doi: https://doi.org/10.1007/s00204-016-1920-7 
6. Mariscal-Arcas M, Lopez-Martinez C, Granada A, Olea N, Lorenzo-Tovar ML, Olea-Serrano F. Organochlorine pesticides in umbilical cord blood serum of women from Southern Spain and adherence to the Mediterranean diet. Food Chem Toxicol 2010, 48 (5): 1311-1315. doi: https://doi.org/10.1016/j.fct.2010.02.029

7. Rauh VA. Polluting Developing Brains - EPA Failure on Chlorpyrifos. N Engl J Med 2018, 378 (13): 1171-1174. doi: https://doi.org/10.1056/NEJMp1716809

8. Liu J, Brannen KC, Grayson DR, Morrow AL, Devaud LL, Lauder JM. Prenatal exposure to the pesticide dieldrin or the $\mathrm{GABA}(\mathrm{A})$ receptor antagonist bicuculline differentially alters expression of GABA(A) receptor subunit mRNAs in fetal rat brainstem. Dev Neurosci 1998, 20 (1): 83-92. doi: https://doi.org/10.1159/000017302

9. Slotkin TA, Seidler FJ. Oxidative and excitatory mechanisms of developmental neurotoxicity: transcriptional profiles for chlorpyrifos, diazinon, dieldrin, and divalent nickel in PC12 cells. Environ Health Perspect 2009, 117 (4): 587-596. doi: https://doi.org/10.1289/ehp.0800251

10. Rani L, Thapa K, Kanojia N, Sharma N, Singh S, Grewal AS, et al. An extensive review on the consequences of chemical pesticides on human health and environment. Journal of Cleaner Production 2021, 283. doi: https://doi.org/10.1016/j.jclepro.2020.124657

11. Tarmure S, Alexescu TG, Orasan O, Negrean V, Sitar-Taut AV, Coste SC, et al. Influence of pesticides on respiratory pathology - A literature review. Annals of Agricultural and Environmental Medicine 2020, 27 (2): 194-200. doi: https://doi.org/10.26444/aaem/121899

12. Kim KH, Kabir E, Jahan SA. Exposure to pesticides and the associated human health effects. Science of the Total Environment 2017, 575: 525-535. doi: https://doi.org/10.1016/j.scitotenv.2016.09.009

13. Genuis SJ, Kelln KL. Toxicant exposure and bioaccumulation: a common and potentially reversible cause of cognitive dysfunction and dementia. Behavioural neurology 2015, 2015: 620143-620143. doi: https://doi.org/10.1155/2015/620143

14. Russo EB. Current Therapeutic Cannabis Controversies and Clinical Trial Design Issues. 2016, 7 (309). doi: https://doi.org/10.3389/fphar.2016.00309

15. Page MJ, McKenzie JE, Bossuyt PM, Boutron I, Hoffmann TC, Mulrow CD, et al. The PRISMA 2020 statement: an updated guideline for reporting systematic reviews. BMJ 2021, 372: n71. doi: https://doi.org/10.1136/bmj.n71

16. Andreo-Martínez P, García-Martínez N, Sánchez-Samper EP, Martínez-González AE. An approach to gut microbiota profile in children with autism spectrum disorder. Environ Microbiol Rep 2019, 12 (2): 115-135. doi: https://doi.org/10.1111/1758-2229.12810

17. Martínez-González AE, Andreo-Martínez P. The Role of Gut Microbiota in Gastrointestinal Symptoms of Children with ASD. Medicina 2019, 55 (8): 408 . doi: https://doi.org/10.3390/medicina55080408

18. Amr MM. Pesticide monitoring and its health problems in Egypt, a Third World country. Toxicol Lett 1999, 107 (1-3): 1-13. doi: https://doi.org/10.1016/s0378-4274(99)00026-0

19. M Khan K, Karnati J, Hamid I, Koceja D, Zahirul Islam M, Khan MA. Residential Proximity to Agricultural Fields and Neurological and Mental Health Outcomes in Rural Adults in Matlab, Bangladesh. International journal of environmental research and public health 2019, 16 (18): 3228. doi: https://doi.org/10.3390/ijerph16183228

20. Yalçın SS, Örün E, Yalçın S, Aykut O. Organochlorine pesticide residues in breast milk and maternal psychopathologies and infant growth from suburban area of Ankara, Turkey. Int J Environ Health Res 2015, 25 (4): 364-372. doi: https://doi.org/10.1080/09603123.2014.945515

21. Zhang J, Liu H, Li J, Lou L, Zhang S, Feng D, et al. Exposure to deltamethrin in adolescent mice induced thyroid dysfunction and behavioral disorders. Chemosphere 2020, 241: 125118. doi: https://doi.org/10.1016/j.chemosphere.2019.125118 
22. Andreo-Martínez P, García-Martínez N, Sánchez-Samper EP, Martínez-González AE. An approach to gut microbiota profile in children with autism spectrum disorder. Environ Microbiol Rep 2020, 12 (2): 115-135. doi: https://doi.org/10.1111/1758-2229.12810

23. Souza RC, Portella RB, Almeida PVNB, Pinto CO, Gubert P, Santos da Silva JD, et al. Human milk contamination by nine organochlorine pesticide residues (OCPs). Journal of Environmental Science and Health - Part B Pesticides, Food Contaminants, and Agricultural Wastes 2020, 55 (6): 530-538. doi: https://doi.org/10.1080/03601234.2020.1729630

24. Li ZM, Albrecht M, Fromme H, Schramm KW, De Angelis M. Persistent Organic Pollutants in Human Breast Milk and Associations with Maternal Thyroid Hormone Homeostasis. Environ Sci Technol 2020, 54 (2): 1111-1119. doi: https://doi.org/10.1021/acs.est.9b06054

25. Lenters V, Iszatt N, Forns J, Čechová E, Kočan A, Legler J, et al. Early-life exposure to persistent organic pollutants (OCPs, PBDEs, PCBs, PFASs) and attention-deficit/hyperactivity disorder: A multipollutant analysis of a Norwegian birth cohort. Environment International 2019, 125: 33-42. doi: https://doi.org/10.1016/j.envint.2019.01.020

26. Richardson JR, Taylor MM, Shalat SL, Guillot TS, 3rd, Caudle WM, Hossain MM, et al. Developmental pesticide exposure reproduces features of attention deficit hyperactivity disorder. Faseb j 2015, 29 (5): 1960-1972. doi: https://doi.org/10.1096/fj.14-260901

27. Kumar A, Sasmal D, Sharma N. Immunomodulatory role of piperine in deltamethrin induced thymic apoptosis and altered immune functions. Environ Toxicol Pharmacol 2015, 39 (2): 504-514. doi: https://doi.org/10.1016/j.etap.2014.12.021

28. Fuhrimann S, Farnham A, Staudacher P, Atuhaire A, Manfioletti T, Niwagaba CB, et al. Exposure to multiple pesticides and neurobehavioral outcomes among smallholder farmers in Uganda. Environment International 2021, 152. doi: https://doi.org/10.1016/j.envint.2021.106477

29. Shrestha S, Parks CG, Umbach DM, Richards-Barber M, Hofmann JN, Chen H, et al. Pesticide use and incident Parkinson's disease in a cohort of farmers and their spouses. Environmental Research 2020, 191. doi: https://doi.org/10.1016/j.envres.2020.110186

30. Risch S, Janowsky DJNomd. Cholinergic-adrenergic balance in affective illness. 1984: 652-663.

31. Coronado GD, Holte S, Vigoren E, Griffith WC, Barr DB, Faustman E, et al. Organophosphate pesticide exposure and residential proximity to nearby fields: evidence for the drift pathway. J Occup Environ Med 2011, 53 (8): 884-891. doi: https://doi.org/10.1097/JOM.0b013e318222f03a 